\title{
POPULAÇÕES, MATRIZES E IDADE DA PLANTA NA EXPRESSÃO DE VARIÁVEIS FÍSICAS EM FRUTOS DO PEQUIZEIRO ${ }^{1}$
}

\author{
FERNANDO HIGINO DE LIMA E SILVA², JOSÉ SEBASTIÃO CUNHA FERNANDES ${ }^{3}$, \\ ELIZABETHE ADRIANA ESTEVES ${ }^{4}$, MIRANDA TITON ${ }^{5}$, REYNALDO CAMPOS SANTANA $^{6}$
}

RESUMO - O pequi é uma espécie com ampla distribuição no cerrado brasileiro, tendo grande importância social e econômica para os habitantes desse bioma. O presente trabalho foi proposto em função da ausência de informações sobre os efeitos de fatores genéticos e ambientais na expressão de variáveis físicas em frutos dessa espécie. Informações dessa natureza facilitam as decisões em relação ao processo de domesticação e melhoramento dessa espécie. Para atender a este objetivo, colheram-se frutos nos municípios de Curvelo e São Gonçalo do Rio Preto, Minas Gerais, totalizando 15 matrizes por município, selecionadas através de características que refletem suas idades, sendo a principal o diâmetro do tronco rente ao solo (DAS). As variáveis físicas avaliadas foram: Peso Total do Fruto (PTF); Peso do Mesocarpo Externo (PME); Peso dos Putamens (PTP) por fruto; $\mathrm{N}^{\circ}$ de Putamens (NP) por fruto e Peso Total de Polpa (PTPL) por fruto. O efeito de matrizes foi altamente significativo para todas as variáveis avaliadas, enquanto o de populações foi apenas para NP. As estimativas das correlações entre as variáveis avaliadas foram todas positivas, algumas significativas. As estimativas das correlações entre DAS e as demais variáveis também foram todas positivas, algumas significativas. Os resultados permitem concluir que: há grandes expectativas de ganhos a partir da propagação vegetativa de matrizes selecionadas no campo para as características físicas de seus frutos; o peso de polpa por fruto, caráter de grande importância econômica, mas de avaliação trabalhosa, pode ser selecionado a partir da avaliação do peso de putamens por fruto; o efeito da idade da planta sobre a expressão de variáveis físicas em frutos de pequi é nulo ou positivo, podendo a seleção ser conduzida em plantas jovens. Termos para indexação: Caryocar brasiliense Camb., variabilidade genética, seleção precoce, correlação fenotípica.

\section{POPULATIONS, MOTHER TREE AND PLANT AGE EFFECTS ON PHYSICAL TRAITS IN PEQUI FRUITS}

\begin{abstract}
Pequi is a very common tree in Central and Center-East Brazilian territory, known as Cerrado sensu strict, and its fruits are largely appreciated by people that live in these regions. This study was proposed due to the lack of information about environmental and genetic effects on physical attributes expression in pequi fruits. Information on this subject is relevant to favor decisions regarding to domestication and breeding processes of this species. Pequi fruits were collected from populations of Curvelo and São Gonçalo do Rio Preto, State of Minas Gerais, being 15 mother trees per population. These mother trees were selected based on physical attributes that imply their age, being stem diameter at ground level (SD), the main one. Physical attributes were: Fruit Total Weight (FTW); Outer Mesocarp Weight (OMW); Putamen Weight per fruit (PW); Putamen Number per fruit (PN) and Pulp Total Weight per fruit (PLTW). There was a mother tree effect for all attributes evaluated, whereas for populations, there was effect only for PN. Correlations among all variables were positive, being some of them significant. Also, correlations between SD and all other variables were positive and some, significant. Results allow the conclusion of high expected gains through vegetative propagation of mother tree selected by means physical fruit attributes: pulp weight can be selected based on putamen weight; the effect of plant age on fruit physical attributes is null or positive, so the selection can be carried out in youth plants.
\end{abstract}

Index terms: Caryocar brasiliense Camb., genetic variability, early selection, phenotypic correlation.

\footnotetext{
${ }^{1}$ (Trabalho 159-11). Recebido em: 27-05-2011. Aceito para publicação em: 28-08-2012.

${ }^{2}$ Eng. Agr., Mestre em Prod. Vegetal - UFVJM, Campus JK, Diamantina-MG. CEP 39100-000. E-mail : fhiginolima@yahoo.com.br ${ }^{3}$ Eng. Agr., Prof. Associado - UFVJM, Campus JK, Diamantina-MG. CEP 39100-000. E-mail: cunha.fernandes@yahoo.com.br ${ }^{4}$ Nutricionista, Prof. Adjunta - UFVJM, Campus JK, Diamantina-MG. CEP 39100-000. E-mail: eaesteves@yahoo.com.br ${ }^{5}$ Eng. Florestal, Prof. Adjunta - UFVJM, Campus JK, Diamantina-MG. CEP 39100-000. E-mail: titonmiranda@yahoo.com.br ${ }^{6}$ Eng. Florestal, Prof.Adjunto - UFVJM, Campus JK, Diamantina-MG. CEP 39100-000. E-mail: silviculturaufvjm@yahoo.com.br
} 


\section{INTRODUÇÃO}

O Cerrado, com mais de $2.000 .000 \mathrm{~km}^{2}$, é o segundo maior bioma brasileiro em extensão, ocupando aproximadamente $23 \%$ do território nacional (QUEIROZ, 2009). Dentre as espécies nativas do Cerrado, chama a atenção o pequizeiro (Caryocar brasiliense Camb.,), que possui várias denominações populares, como piqui, pequiá, amêndoa-de- espinho, grão-de-cavalo-pequi, amêndoa-do-brasil e pequi, sendo este o mais comumente utilizado.

No norte de Minas Gerais, sua ocorrência é comum, mobilizando $50 \%$ da população que vive no campo, em épocas de colheita (ALENCAR, 2000). Os frutos de pequi assumem um papel importante na vida dos sertanejos, seja com a venda do fruto in natura, seja mesmo para seu próprio consumo (MARIANO et al., 2009), sendo bastante utilizados no preparo de pratos típicos, condimentos, óleos e bebidas adocicadas. Entretanto, a futura exploração sustentável dessa atividade encontra-se ameaçada por limitações como: erosão genética, domesticação da espécie, complexidade das técnicas de propagação e inexistência de padrões de qualidade na comercialização.

O pequizeiro geralmente cresce como uma árvore frondosa, esgalhada e com altura que pode ultrapassar os 15 metros no Cerrado de Minas Gerais. O fruto caracteriza-se por ser drupoide, verde, com epicarpo coriáceo carnoso, contendo de um a quatro caroços (putamens ou pirênios) envolvidos por um mesocarpo amarelo-claro, endocarpo lenhoso espinhoso e sementes reniformes (ALMEIDA et al., 1998). O pequizeiro caracteriza-se como uma planta alógama com dispersão tipicamente zoocórica (MELO JR. et al., 2004), no entanto sua propagação e cultivo têm sido dificultados pela baixa e lenta germinação das sementes que se estende por período de até um ano (ROCHA, 2009). Uma alternativa existente, ainda mesmo que incipiente, é a utilização de métodos de propagação vegetativa, em que o emprego da enxertia constitui-se no mais utilizado na produção de mudas de pequizeiro.

Pelo fato de ser uma espécie não domesticada, observa-se grande variação entre matrizes para algumas características físicas nos frutos, como tamanho, coloração, número de putamens por fruto, peso de putamens e de suas respectivas polpas. Deste modo, a avaliação destas variáveis em diferentes matrizes de diferentes populações é importante para seu melhoramento. Porém, quando se trabalha com espécies perenes, como é o caso do pequizeiro, o tempo para se completar um ciclo de seleção é a principal limitação dos programas de melhoramento genético.
Dessa forma, devem-se utilizar alternativas que visem a diminuir o tempo necessário para completar um ciclo de seleção, ou seja, promover a seleção na idade mais juvenil possível (PEREIRA et al., 1997). Entretanto, a seleção em idade juvenil só é viável se houver garantia de que o caráter selecionado nesta idade terá expressão equivalente na idade adulta.

Este trabalho teve por objetivo avaliar os efeitos de populações, matrizes e idade na planta, na expressão de características físicas, em frutos de pequizeiros oriundos de duas procedências no Estado de Minas Gerais.

\section{MATERIAL E MÉTODOS}

Foram amostradas matrizes de populações provenientes do Parque Estadual de Rio Preto, município de São Gonçalo do Rio Preto-MG (latitude $18^{\circ}$ 07’2.6”, longitude $43^{\circ} 20^{\prime}$ '51.7”' e altitude média de 750 m) e da Estação Experimental do Moura, da Universidade Federal do Vale do Jequitinhonha e Murici (UFVJM), município de Curvelo-MG (latitude $18^{\circ}$ $45^{\prime}$ '23”, longitude $44^{\circ} 25^{\prime} 51^{\prime \prime}$ e altitude média de $650 \mathrm{~m}$ ). Em função da distância geográfica entre estes dois locais, que é de aproximadamente $200 \mathrm{~km}$, suas populações podem ser consideradas isoladas entre si, reprodutivamente.

A coleta, em ambos os locais, ocorreu durante o mês de janeiro do ano de 2010. Os frutos foram coletados no estágio de maturação completa (frutos caídos no chão). Para se proceder às avaliações físicas, foram amostrados 30 frutos por matriz e 15 matrizes por local.

As matrizes foram previamente escolhidas, levando-se em consideração características que refletem suas idades, sendo as principais o diâmetro do tronco rente ao solo (DAS) e a rugosidade da casca. Matrizes com DAS variando entre 10 e $20 \mathrm{~cm}$ e com casca lisa são seguramente matrizes novas, e aquelas com DAS acima de $40 \mathrm{~cm}$ são matrizes velhas. Já que não se pode inferir a idade a partir do DAS, esta última foi tomada como uma estimativa da magnitude da idade.

Logo após a coleta, os frutos foram devidamente acondicionados para evitar danos mecânicos e, em seguida, transportados para o Laboratório Integrado de Pesquisas Multiusuário dos Vales do Jequitinhonha e Mucuri (LIPEMVALE), da UFVJM, onde ficaram armazenados em refrigerador, por três dias, até a realização dos ensaios analíticos.

As variáveis físicas avaliadas foram: Peso Total do Fruto (PTF); Peso do Mesocarpo Externo por fruto (PME); Peso dos Putamens por fruto (PTP); Número de Putamens por fruto (NP) e Peso Total de 
Polpa por fruto (PTPL). Outras variáveis derivadas destas também foram computadas, com Peso Médio de Polpa por Putamen (PMPL) e Peso Médio de Putamens por fruto (PMP). As variáveis de massa foram obtidas com auxílio de uma balança digital semianalítica com precisão de 0,001g, marca Shimadzu.

A unidade amostral para a avaliação destas variáveis foi composta por um fruto, e de cada matriz foram avaliados 10 frutos tomados aleatoriamente.

Os dados foram analisados seguindo uma classificação hierárquica a partir do modelo: $\mathrm{Y}_{\mathrm{ijk}}=\mathrm{m}$ $+\mathrm{P}_{\mathrm{i}}+\mathrm{M}_{\mathrm{j} / \mathrm{i}}+\mathrm{F}_{\mathrm{k} / \mathrm{j}}$, em que $\mathrm{m}=$ média geral; $\mathrm{P}_{\mathrm{i}}=$ efeito da população i; $\mathrm{M}_{\mathrm{j} / \mathrm{i}}=$ efeito da matriz $\mathrm{j}$ dentro da população i; e $\mathrm{F}_{\mathrm{k} / \mathrm{j}}$ é o efeito do fruto $\mathrm{k}$ dentro da matriz j, dentro da população i. As hipóteses dos efeitos do modelo para cada variável, exceto o diâmetro do tronco, foram testadas através do teste $\mathrm{F}$.

A hipótese do efeito da idade sobre as variáveis físicas foi testada através da estimativa do coeficiente de correlação (r) entre o valor médio de cada variável por matriz com o diâmetro (variável usada para inferir a idade) da respectiva matriz, sendo esta testada através do teste t de student. Foi utilizado o Software Excel 2007 para a análise estatística dos dados.

\section{RESULTADOS E DISCUSSÃO}

Para todas as variáveis físicas, a análise de variância mostrou diferenças altamente significativas $(P<0,01)$ entre matrizes, enquanto, para populações, houve significância $(\mathrm{P}=5,47 \%)$ apenas para NP (Tabela 01).

Para o peso total do fruto (PTF), foi observada variação de 83,05 a 259,98 g entre as médias das matrizes avaliadas nas duas populações (Tabela 02). Correa et al. (2008) observaram variação de 46,93 a $165,16 \mathrm{~g}$ entre frutos de pequizeiros provenientes de diferentes municípios do Estado de Goiás. Oliveira et al. (2009), para a espécie Caryocar coriaceum, reportaram variação entre as médias das matrizes de 46,26 a 140,23 g, oriundas da Chapada do Araripe, sul do Estado do Ceará.

Para o peso do mesocarpo externo (PME), as médias entre matrizes variaram de 61,57 a 220,00 g (Tabela 02). Vera et al. (2007) encontraram, para essa variável, amostrando frutos de dois municípios do Estado de Goiás, variação de 59,32 a 303,80 g para a população de Mambaí e 34,54 a 174,32 g para Araguapaz.

É interessante observar que, embora a amplitude de variação observada entre as médias de matrizes para PTF e PME seja alta, os valores mínimo e máximo para a razão PME/PTF foram 0,67 (matriz
1) e 0,87 (matriz 8), respectivamente, observando-se média para essa relação, nas duas populações, de 0,78, sendo esses valores obtidos a partir da Tabela 02. Infere-se que aproximadamente três quartos do peso do fruto é determinado pelo peso do mesocarpo externo, sendo esta também uma justificativa para a alta correlação positiva $(0,98)$ observada entre estas duas variáveis (Tabela 03). Este aspecto foi enfatizado também por Vera et al. (2005) ao observarem que o peso da casca representou 76,72\% da massa total do fruto em 75 matrizes oriundas de cinco regiões do Estado de Goiás. Vale lembrar que, embora a casca do pequi seja considerada um resíduo, não sendo aproveitada comercialmente até o momento (VERA et al., 2007), existem estudos sobre a viabilidade de seu uso na alimentação animal.

As médias por matriz para a variável NP oscilaram de 1,00 a 2,20 (Tabela 02). Correa et al. (2008) observaram, para o número de putamens, variação entre frutos de 1,08 a 2,50. Silva et al. (2001) observaram maior incidência de frutos com apenas um putâmen desenvolvido. Essa foi também a variável em que se observou maior diferença entre as duas populações (Tabela 01). Para o consumo in natura, buscam-se frutos com polpa de putamens de maior espessura, e não somente a quantidade de putamens por fruto. No entanto, maior quantidade de putamens por frutos seria importante do ponto de vista prático quando se tem por objetivo a exploração econômica da amêndoa do pequi, que é de grande aceitação no mercado devido ao seu elevado teor de proteínas.

As médias por matriz para a variável PTP oscilaram entre 18,29 e 54,37 g (Tabela 02). Vera (2004) observou, para essa variável, variação entre frutos de 7,77 a 16,10 g, avaliando pequizeiros oriundos de diferentes populações do Estado de Goiás. Vera et al. (2007) observaram efeito significativo de populações na variável peso total de putamens para frutos oriundos das regiões de Araguapaz e Mambaí, também no Estado de Goiás. Porém, neste último trabalho, para as variáveis avaliadas, não se objetivou a determinação do efeito de matrizes. Correa et al. (2008), coletando frutos de um total de 35 matrizes, em três municípios do Estado de Goiás, observaram médias por matriz entre 32,49 e 138,37 g. Para o peso médio dos putamens (PMP), os valores médios por matriz observados oscilaram entre 13,03 e 32,71 g (Tabela 02). Do ponto de vista comercial, essa variável assume grande importância, pois frutos com baixo rendimento de polpa e amêndoa podem inviabilizar o processo de industrialização (VERA et al., 2005).

Para a variável PTPL, as médias entre matrizes variaram de 7,15 a 25,27 g (Tabela 02). Vera 
et al. (2007) observaram, também para essa variável, efeito significativo de populações, sendo os valores médios de 3,98 g para a região de Araguapaz e 3,31 g para Mambaí. Para a espécie Caryocar coriaceum, Oliveira et al. (2009) observaram variação de 5,11 a 19,62 g entre matrizes oriundas do Estado do Ceará. A massa total de polpa é uma característica física de grande importância econômica, pois é a parte do pequi mais consumida na alimentação humana e, por esse motivo, desperta maior interesse comercial (VERA et al., 2007).

Os valores médios por matriz para a variável PMPL (Tabela 02) variaram entre 5,38 e 14,25 g. Para essa variável, Souza (2005) observou variação de 3,56 a 12,65 g entre frutos de pequizeiros de duas populações diferentes, também no Estado de Goiás. Em um programa de seleção visando à maior quantidade de polpa por frutos, devem-se priorizar frutos com maior peso total de polpa em vez do peso médio de polpa por putamens.

Variações entre matrizes dentro de populações são comumente observadas para variáveis em espécies como Araucaria angustifolia (SHIMIZU et al., 1999), Acrocomia aculeata (OLIVEIRA et al., 2008a), Eremanthus erythropappus MacLeish (ESTOPA et al., 2006), Dimorphandra mollis Benth (OLIVEIRA et al., 2008b), Dipteryx alata (SIQUEIRA et al., 1993) e Tabebuia vellosoi $T$. (ETTORI et al., 1995). Espécies arbóreas, em geral, mostram maior variação genética dentro de populações. No presente trabalho, maiores níveis de variação entre matrizes, para todas as variáveis, exceto NP, foram observados dentro das populações (Tabela 01). No caso do pequizeiro, Vilela (1998) reportou que a baixa divergência entre populações pode ser devida ao alto fluxo gênico entre estas, decorrente do sistema de polinização da espécie, que é realizada principalmente pelo morcego, cujo voo atinge grandes distâncias, e também à dispersão das sementes, que é realizada por grandes aves e alguns mamíferos. Outra hipótese considerada é a possibilidade de populações distantes serem resultantes da fragmentação de uma única população há um tempo não suficiente para causar divergências entre estas.

As estimativas dos coeficientes de correlação para NP e demais variáveis físicas foram, em sua maioria, significativas a $5 \%$ de probabilidade (Tabela 03). Apenas duas estimativas foram negativas, mas não significativas: NP x PMP e NP x PMPL. A correlação negativa não causa surpresa uma vez que se espera que frutos com mais putamens os têm menores e, consequentemente, com menor quantidade de polpa.

A correlação entre caracteres, um parâmetro estatístico que mede o grau de associação entre duas variáveis, é importante do ponto de vista do melhoramento (ALMEIDA et al., 2010). Tal informação permite ao melhorista o conhecimento das mudanças que ocorrem em um caráter quando se realiza a seleção em outro caráter a ele correlacionado. Em um programa de melhoramento genético, que visa à seleção de materiais com características adequadas à comercialização e ao processamento, informações neste sentido devem ser consideradas.

No presente estudo, é pertinente a observação das estimativas das correlações entre as variáveis avaliadas e a variável PTPL que, por motivos já apresentados, é a de maior interesse comercial. Observa-se que a variável PTP é a que exibe maior correlação com PTPL (Tabela 03). Considerando-se que a retirada da polpa é um trabalho extremamente laborioso, a variável PTP pode e deve ser usada para se selecionar frutos com alta quantidade de polpa. A seleção em PTF não seria recomendada dada a sua baixa correlação com PTPL (Tabela 03).

As estimativas de correlações entre DAS e as características físicas (Tabela 04) mostram que, por serem todas positivas, há uma tendência de aumento do valor das variáveis em estudo com o aumento do DAS. O fato de estas correlações serem não significativas e positivas é um indicativo de que a seleção de matrizes com características desejáveis pode ser feita em plantas jovens.

Embora não se possa descartar a possibilidade de efeitos do ambiente na variação observada entre as matrizes, inclusive suas idades, conforme evidenciado por algumas correlações significativas entre o DAS e as variáveis estudadas, há fortes evidências de que a maior parte desta variação decorre das diferenças genéticas entre estas. Considerando-se que todas as variáveis em estudo foram feitas em tecidos maternos, é de se esperar, também, que as diferenças genéticas observadas entre as matrizes sejam efeitos maternos e não decorrentes das diferenças entre os genótipos das sementes (efeito xênia). Aceitando-se a hipótese da predominância dos efeitos maternos, ganhos estimados com a seleção, visando à produção de mudas, só fariam sentido se os indivíduos da população melhorada possuíssem os mesmos genótipos dos indivíduos da população selecionada, ou seja, se obtidos por propagação vegetativa. Segundo Ramalho et al. (2008), o efeito materno é um caso especial de herança controlado por genes nucleares da mãe, sendo responsáveis por certas condições do citoplasma do óvulo, provavelmente produtos gênicos. O conhecimento desse tipo de herança em frutíferas, principalmente espécies nativas, como Caryocar brasilense Camb., é bastante escasso. 
TABELA 1 - Probabilidades de significância P(F) dos efeitos de populações e matrizes dentro de populações sobre algumas variáveis em frutos de pequizeiro (Caryocar brasiliense Camb.) oriundos dos municípios de Curvelo e São Gonçalo do Rio Preto, Minas Gerais.

\begin{tabular}{|c|c|c|c|c|c|c|c|c|}
\hline \multirow{3}{*}{ FV } & \multirow{3}{*}{ GL } & \multicolumn{7}{|c|}{ Probabilidade F (\%) } \\
\hline & & \multicolumn{7}{|c|}{ Variáveis $^{1}$} \\
\hline & & PTF & PME & NP & PTP & PMP & PTPL & PMPL \\
\hline Populações & 1 & 43,29 & 30,05 & 5,47 & 51,22 & 50,23 & 29,48 & 89,74 \\
\hline Matrizes / Populações & 28 & 0,00 & 0,00 & 0,30 & 0,00 & 0,00 & 0,00 & 0,00 \\
\hline Frutos/Matrizes/Populações & 270 & & & & & & & \\
\hline
\end{tabular}

${ }^{1}$ PTF: peso total do fruto; PME: peso do mesocarpo externo; NP: número de putamens; PTP: peso total de putamens; PMP: peso médio de putamens; PTPL: peso total de polpa; PMPL: peso médio de polpa.

TABELA 2- Valores médios, para cada variável ${ }^{1}$, de matrizes de pequizeiro oriundas de Curvelo e São Gonçalo do Rio Preto, Minas Gerais.

\begin{tabular}{|c|c|c|c|c|c|c|c|c|c|}
\hline População & Matriz & DAS & PTF & PME & NP & PTP & PMP & PTPL & PMPL \\
\hline Curvelo & 1 & 41,1 & 91,95 & 61,57 & 1,60 & 30,38 & 19,38 & 13,69 & 8,68 \\
\hline Curvelo & 4 & 29,0 & 98,50 & 74,50 & 1,30 & 24,01 & 19,00 & 10,00 & 7,93 \\
\hline Curvelo & 6 & 30,6 & 148,06 & 119,93 & 1,30 & 28,13 & 22,55 & 11,99 & 9,65 \\
\hline Curvelo & 8 & 42,0 & 178,98 & 156,46 & 1,30 & 22,52 & 17,54 & 8,47 & 6,68 \\
\hline Curvelo & 11 & 43,9 & 128,13 & 104,62 & 1,20 & 23,52 & 19,35 & 10,51 & 8,65 \\
\hline Curvelo & 15 & 34,4 & 110,08 & 91,72 & 1,30 & 18,36 & 14,94 & 7,15 & 5,77 \\
\hline Curvelo & 17 & 54,8 & 184,36 & 155,02 & 1,30 & 29,34 & 23,10 & 13,76 & 11,11 \\
\hline Curvelo & N02 & 21,0 & 166,60 & 124,19 & 1,70 & 42,41 & 25,55 & 19,77 & 11,67 \\
\hline Curvelo & N03 & 19,1 & 143,24 & 118,31 & 1,70 & 24,94 & 15,06 & 10,35 & 6,35 \\
\hline Curvelo & N04 & 41,4 & 226,80 & 183,49 & 1,50 & 43,31 & 28,73 & 21,64 & 14,25 \\
\hline Curvelo & N05 & 23,9 & 259,98 & 220,00 & 2,00 & 39,98 & 20,53 & 14,36 & 7,11 \\
\hline Curvelo & N07 & 15,3 & 101,83 & 72,54 & 1,00 & 29,28 & 29,28 & 11,75 & 11,75 \\
\hline Curvelo & N08 & 44,6 & 218,20 & 173,13 & 1,40 & 45,07 & 32,71 & 16,23 & 11,62 \\
\hline Curvelo & N09 & 27,4 & 154,60 & 125,76 & 1,30 & 28,84 & 23,13 & 10,72 & 8,52 \\
\hline \multirow[t]{2}{*}{ Curvelo } & N10 & 33,1 & 104,82 & 78,86 & 1,40 & 25,95 & 20,30 & 9,61 & 7,44 \\
\hline & & Média & 154,41 & 124,01 & 1,42 & 30,40 & 22,08 & 12,67 & 9,15 \\
\hline Rio Preto & 151 & 41,4 & 189,49 & 135,12 & 1,90 & 54,37 & 28,96 & 25,27 & 13,55 \\
\hline Rio Preto & 167 & 55,1 & 193,06 & 158,47 & 2,20 & 34,59 & 16,66 & 13,12 & 6,35 \\
\hline Rio Preto & 168 & 29,3 & 177,22 & 146,95 & 2,10 & 30,27 & 14,22 & 14,59 & 6,87 \\
\hline Rio Preto & 172 & 29,6 & 135,47 & 103,39 & 1,90 & 32,08 & 17,59 & 14,29 & 7,85 \\
\hline Rio Preto & 174 & 44,6 & 151,98 & 120,83 & 1,80 & 31,16 & 18,67 & 11,57 & 6,92 \\
\hline Rio Preto & N151 & 20,4 & 128,91 & 94,01 & 1,30 & 34,90 & 27,46 & 15,45 & 12,12 \\
\hline Rio Preto & N152 & 22,3 & 151,30 & 114,68 & 1,20 & 36,62 & 30,06 & 16,24 & 13,39 \\
\hline Rio Preto & N153 & 16,9 & 155,83 & 117,07 & 1,80 & 38,76 & 22,44 & 21,10 & 12,23 \\
\hline Rio Preto & N154 & 15,3 & 144,63 & 117,82 & 1,20 & 26,81 & 23,36 & 12,44 & 10,87 \\
\hline Rio Preto & N155 & 15,6 & 83,05 & 62,58 & 1,20 & 20,48 & 17,50 & 9,79 & 8,38 \\
\hline Rio Preto & N156 & 14,0 & 118,84 & 100,55 & 1,40 & 18,29 & 13,03 & 7,50 & 5,38 \\
\hline Rio Preto & N157 & 12,7 & 116,30 & 90,44 & 1,80 & 25,87 & 15,21 & 12,50 & 7,72 \\
\hline Rio Preto & N158 & 13,4 & 105,15 & 75,05 & 1,50 & 30,10 & 19,06 & 11,41 & 7,70 \\
\hline Rio Preto & N159 & 43,6 & 145,97 & 110,33 & 1,70 & 35,64 & 21,79 & 12,83 & 8,00 \\
\hline \multirow[t]{3}{*}{ Rio Preto } & N160 & 37,6 & 134,82 & 98,12 & 1,50 & 36,70 & 25,46 & 16,59 & 11,69 \\
\hline & & Média & 142,13 & 109,69 & 1,63 & 32,44 & 20,76 & 14,31 & 9,27 \\
\hline & & DMS (Tukey 5\%) & 69,83 & 53,05 & 0,99 & 19,72 & 6,42 & 8,59 & 3,23 \\
\hline
\end{tabular}

${ }^{1}$ PTF: peso total do fruto (g); PME: peso do mesocarpo externo (g); NP: número de putamens; PTP: peso total de putamens (g); PMP: peso médio de putamens (g); PTPL: peso total de polpa (g); PMPL: peso médio de polpa (g).

DMS (Diferença mínima significativa): Referem-se à comparação entre matrizes dentro de cada população. 
TABELA 3 - Estimativas dos coeficientes de correlação fenotípica para as variáveis ${ }^{1}$ físicas, avaliadas em frutos de pequizeiro (Caryocar brasiliense Camb.), oriundos dos municípios de Curvelo e São Gonçalo do Rio Preto, Minas Gerais.

\begin{tabular}{cccccccc}
\hline & PTF & PME & NP & PTP & PMP & PTPL & PMPL \\
\hline PTF1 & - & $0,9850^{* *}$ & $0,4359^{*}$ & $0,6369^{* *}$ & $0,3318 \mathrm{~ns}$ & $0,5103^{* *}$ & $0,2526 \mathrm{~ns}$ \\
PME & & - & $0,3973^{*}$ & $0,4944^{* *}$ & $0,2158 \mathrm{~ns}$ & $0,3703^{*}$ & $0,1324 \mathrm{~ns}$ \\
NP & & & - & $0,4213^{*}$ & $-0,3141 \mathrm{~ns}$ & $0,3796^{*}$ & $-0,2474 \mathrm{~ns}$ \\
PTP & & & & - & $0,7077^{* *}$ & $0,9167^{* *}$ & $0,6810^{* *}$ \\
PMP & & & & & - & $0,6372^{* *}$ & $0,8984^{* *}$ \\
PTPL & & & & & & - & $0,7843^{* *}$ \\
PMPL & & & & & & - \\
\hline
\end{tabular}

${ }^{1}$ PTF: peso total do fruto; PME: peso do mesocarpo externo; NP: número de putamens; PTP: peso total de putamens; PMP: peso médio de putamens; PTPL: peso total de polpa; PMPL: peso médio de polpa.

$\mathrm{ns},{ }^{*} \mathrm{e} * *$ : não significativo, significativo a $5 \%$ e $1 \%$, respectivamente, pelo teste $\mathrm{F}$.

TABELA 4- Estimativas dos coeficientes de correlação (r) entre o diâmetro rente ao solo (DAS) e as variáveis ${ }^{1}$ físicas, e suas respectivas probabilidades de significância $\mathrm{P}(\mathrm{t})$, para matrizes de Caryocar brasiliense Camb., oriundas dos municípios de Curvelo e São Gonçalo do Rio Preto, Minas Gerais.

\begin{tabular}{lcc}
\hline Variáveis & $\mathbf{r}$ & $\mathbf{P ( t )}$ \\
\hline PTF & 0,3935 & 2,87 \\
PME & 0,3950 & 3,08 \\
NP & 0,1911 & 31,17 \\
PTP & 0,2479 & 18,66 \\
PMP & 0,1132 & 55,14 \\
PTPL & 0,1250 & 51,06 \\
PMPL & 0,1190 & 95,02 \\
\hline
\end{tabular}

${ }^{1}$ PTF: peso total do fruto; PME: peso do mesocarpo externo; NP: número de putamens; PTP: peso total de putamens; PMP: peso médio de putamens; PTPL: peso total de polpa; PMPL: peso médio de polpa.

\section{CONCLUSÕES}

1-Há grandes expectativas de ganhos a partir da propagação vegetativa de matrizes selecionadas no campo pelas características físicas de seus frutos.

2-O peso de polpa por fruto, caráter de grande importância econômica, mas de avaliação trabalhosa, pode ser selecionado a partir da avaliação do peso de putamens por fruto devido à alta correlação positiva observada.

3-Considerando-se a magnitude das diferenças entre os diâmetros dos troncos das matrizes amostradas, estes diâmetros têm grande reflexo sobre suas respectivas idades, inferindo-se daí que o efeito da idade da planta sobre a expressão de variáveis físicas em frutos de pequi é nulo ou positivo, podendo a seleção ser conduzida em plantas jovens.

\section{AGRADECIMENTOS}

À Coordenação de Aperfeiçoamento de Pessoal de Nível Superior (CAPES), pela concessão de bolsa de mestrado, e à UFVJM, pelo apoio.

\section{REFERÊNCIAS}

ALENCAR, G. Pequizeiros enfrentam riscos de extinção. Hoje em Dia, Belo Horizonte, 13 fev., 2000. p.7

ALMEIDA, R. D.; PELUZIO, J. M.; AFFERI, F. S. Correlações fenotípicas, genotípicas e ambientais em soja cultivada sob condições de várzea irrigada, sul do Tocantins. Bioscience Journal, Uberlândia, v.26, n.1, p.95-99, 2010. 
ALMEIDA, S. P.; PROENÇA, C. E. B.; SANO, S. M.; RIBEIRO, J. F. Cerrado: espécies vegetais úteis. Planaltina: EMBRAPA-CPAC, 1998. 464p.

CORREA, G. C.; NAVES,R. V.; ROCHA, M. R.; CHAVES, L. J.; BORGES, J. D. Determinações físicas em frutos e sementes de baru (Dipteryx alata Vog.), cajuzinho (Anacardium othonianum Rizz.) e pequi (Caryocar brasiliense Camb.), visando ao melhoramento genético. Bioscience Journal, Uberlândia, v.24, n.4, p.42-47, 2008.

ESTOPA, R. A.; SOUZA. A. M. de; MOURA, M. C. O.; BOTREL, M. C. G.; MENDONÇA,E. G.; CARVALHO, D. Diversidade genética em populações naturais de candeia (Eremanthus erythropappus (DC.) MacLeish). Scientia Forestalis, Piracicaba, n.70, p.97-106, 2006.

ETTORI, L. C.; SIQUEIRA, A. C. M. F.; SATO, A. S.; CAMPOS, O. R. Variabilidade genética em populações de ipê-roxo - Tabebuia heptaphylla (Vell.) Tol. - para conservação ex situ. Revista do Instituto Florestal, São Paulo, v.8, n.1, p.61-70, 1996.

MARIANO, R. G. B.; COURI, S.; FREITAS, S. P. Enzymatic technology to improve oil extraction from Caryocar brasiliense Cam. (pequi) pulp. Revista Brasileira de Fruticultura, Jaboticabal, v. 31, n. 3, p. 637-643, 2009.

MELO JR., A. F. de; CARVALHO, D. de; POVOA, J. S. R.; BEARZOLI, E. Estrutura genética de populações naturais de pequizeiro (Caryocar brasiliense Camb). Scientia Forestalis, Piracicaba, v. 66, p. 56-65, 2004.

OLIVEIRA; D. A.; MELO JÚNIOR, A. F.; BRANDÃO, M. M.; RODRIGUES, L. A.; FONSECA, F. S. A.; FERREIRA, M. F. M.; SILVA, G. M. Diversidade genética de populações de Acrocomia aculeata (Jacq.) Lodd. (Arecaceae) no norte do Estado de Minas Gerais. In: SIMPÓSIO INTERNACIONAL DE SAVANAS TROPICAIS, 2., 2008a, Brasília. Anais... 2008a.

OLIVEIRA, D. A. de; PAULA, M. F. B. de; PIMENTA, M. A. S.; BRAGA, R. F.; FERREIRA, M. F. M.; RODRIGUES, L. A. Variabilidade genética de populações de fava- d' anta (Dimorphandra mollis) da região norte de Minas Gerais. Revista Árvore, Viçosa, MG, v.32, n.2, p.355-363, 2008b.
OLIVEIRA, M. E. B.; GUERRA, N. B.; MAIA, A. H. N.; ALVES, R. E.; XAVIER, D. S.; MATOS, N. M. S. Caracterização física de frutos do pequizeiro nativos da chapada do Araripe-CE. Revista Brasileira de Fruticultura, Jaboticabal, v.31, n.4, 2009.

PEREIRA, A. B.; MARQUES JÚNIOR, O. G.; RAMALHO, M. A. P.; ALTHOFT, P. Eficiência da seleção precoce em famílias de meios-irmãos de Eucalyptus camaldulensis Dehnh., avaliadas na região noroeste do Estado de Minas Gerais. Revista Cerne, Lavras, v.3, n.1, p.67-81, 1997.

QUEIROZ, F. A. Impactos da sojicultura de exportação sobre a biodiversidade do Cerrado. Sociedade \& Natureza, Uberlândia, v.21, n.2, p.193-209, 2009.

RAMALHO, M. A. P.; SANTOS, J. B. dos; PINTO, C. A. B. Genética na agropecuária. 4.ed. Lavras: Editora UFLA, 2008. 464p.

RATTER, J. A.; RIBEIRO, J. F.; BRIDGEWATER, S. The Brazilian Cerrado vegetation and threats to its biodiversity. Annals of Botany, Oxford, v.80, p.223-230, 1997.

ROCHA, J. P. Fatores Genéticos e Ambientais na Emergência de Plântulas de Pequizeiro (Caryocar brasiliense Camb.). 2009. 45 f. Dissertação (Mestrado em Produção Vegetal) - Universidade Federal dos Vales do Jequitinhonha e Mucuri, Diamantina, 2009.

SHIMIZU, J. Y. Variação entre procedências de araucária em Ribeirão Branco (SP) aos vinte e três anos de idade. Boletim de Pesquisa Florestal, Colombo, n.38, p.89-102, 1999.

SILVA, D. B.; SILVA, J. A.; JUNQUEIRA, N. T. V.; ANDRADE, L. R. M. Frutas do cerrado. Brasília: EMBRAPA, 2001. 178p. (Informação Tecnológica) SIQUEIRA, C. M. F.; NOGUEIRA, J. C. B.; KAGEYAMA, P. Y. Conservação dos recursos genéticos ex situ do cumbaru Dipteryx alata Vog.Leguminosae. Revista do Instituto Florestal, São Paulo, v.5, n.2, p.231-243, 1993. 
SOUZA, O. O. A. Caracterização física de frutos e propagação sexuada de pequizeiro (Caryocar brasiliense Camb.) oriundos de diferentes regiões do Estado de Goiás. Estrutura do fruto e da semente do pequi, Caryocar brasiliense Camb. (Caryocaraceae). 2005. 57 f. Dissertação (Mestrado em Agronomia) - Universidade Federal de Goiás, Goiânia, 2005.

VERA, R. Caracterização física e química de frutos do pequizeiro (Caryocar brasiliense Camb.) de diferentes regiões do Estado de Goiás. 2004. 50 f. Dissertação (Mestrado em Agronomia) Universidade Federal de Goiás, Goiânia, 2004.

VERA, R.; NAVES, R. V.; NASCIMENTO, J. L. DO; CHAVES, L. J.; LENDRO, W. M.; SOUZA, E. R. B. de. Caracterização física de frutos do pequizeiro (Caryocar brasiliense Camb.) no Estado de Goiás. Pesquisa Agropecuária Tropical, Goiânia, v.35, p.71-79, 2005.
VERA, R.; SOUZA, E. R. B. DE; FERNANDES, E. P.; NAVES, R. V.; SOARES JÚNIOR, M. S.; CALIARI, M.; XIMENES, P. A. Caracterização física e química de frutos do pequizeiro (Caryocar brasiliense Camb.) oriundos de duas regiões no Estado de Goiás, Brasil. Pesquisa Agropecuária Tropical, Goiânia, v.37, p.93-99, 2007.

VILELA, G. F. Variações em populações naturais de Caryocar brasiliense Camb. (Caryocaraceae): fenológicas, genéticas e de valores nutricionais de frutos. 1998. 88 f. Dissertação (Mestrado em Engenharia Florestal) - Universidade Federal de Lavras, Lavras, 1998. 